\title{
Profesionalización de promotores de lectura con el aprendizaje basado en proyectos mediado por TIC
}

\section{Professionalisation of reading promoters with ICT-mediated project- based learning}

\author{
Mario Miguel Ojeda-Ramírez \\ Universidad Veracruzana. Xalapa. México \\ mojeda@uv.mx \\ Antonia Olivia Jarvio-Fernández \\ Universidad Veracruzana. Xalapa. México \\ ojarvio@uv.mx
}

\begin{abstract}
Resumen
Se revisa brevemente el aprendizaje basado en proyectos (ABP) mediado por TIC con el fin de motivar el contexto adecuado y los fundamentos para presentar el plan de estudios y las estrategias de aprendizaje utilizadas en la Especialización en Promoción de la Lectura (EPL), un programa de posgrado de la Universidad Veracruzana. Se describe el uso de EMINUS, un entorno virtual de aprendizaje institucional, bajo un enfoque bimodal o blended learning; destacando el uso de la bitácora y los resultados asociados con el diseño, la ejecución y el reporte de los proyectos de promoción de la lectura. Se discuten los resultados de las dos primeras generaciones; por último, se identifican las áreas de oportunidad de mejora de este sistema de entrenamiento basado en un esquema de aprendizaje personalizado mediado por TIC.
\end{abstract}

\section{Palabras clave}

Enfoque bimodal, Educación mediada por TIC, Aprendizaje significativo, Estrategias de aprendizaje, Aprendizaje basado en proyectos, Promoción de la lectura.

\begin{abstract}
We briefly reviewiew ICT-mediated project-based learning (BPA) to motivate the appropriate context and the foundations for the presentation of the curriculum and learning strategies used in the Specialization in Reading Promotion (EPL), a postgraduate program at the Universidad Veracruzana. The use of EMINUS, a virtual institutional learning environment, under a bimodal or blended learning approach is described, highlighting the use of the logbook and the results associated with the design, execution and reporting of reading promotion projects. The results of the first two generations are discussed; Finally, we identify the areas of opportunity for improvement of this training system based on a personalized learning scheme mediated by ICT.
\end{abstract}

\section{Key words}

Blended learning, ICT mediated education, Significant learning, Learning strategies, Project based learning, Reading promotion.

\section{Introducción}

Los educadores que vienen trabajando desde las dos últimas décadas del siglo pasado saben muy bien que los sistemas educativos tienen una gran cantidad de resistencias a la innovación y que los cambios, aun cuando se saben necesarios, toman su tiempo, que es el tiempo de una suerte de apropiación cultural que una innovación en particular 
requiere. En este sentido, se puede afirmar que la educación estrictamente presencial se ha quedado en el pasado, ciertamente, porque las opciones de la educación a distancia vienen de mucho tiempo atrás, incluso antes de la irrupción tecnológica. Por otro lado, el uso de la tecnología en la educación aparece como una promesa, que podría decirse que es también antigua, pero toma preeminencia a partir de la última década del siglo XX. Muchos de estos educadores, pero sobre todo los ideólogos de la innovación educativa, se han abrazado a esta promesa, en ocasiones generando quimeras; no obstante, la realidad siempre se ha impuesto, dando los elementos para plantear visiones más verosímiles, propuestas más razonadas. Podemos decir, en este contexto, que la sociedad está cambiando, que la educación está cambiando, pero las promesas -como la de las TIC en la educación- deberán tomarse no como dádivas, sino como caminos posibles, para grupos posibles, donde la diversidad de las circunstancias plantea diversos niveles de garantía de éxito (Tapscott, 2009; Ramírez-Martinell \& CasillasAlvarado, 2014).

Para quienes han vivido el tránsito que va desde el advenimiento de las TIC hasta su incorporación en las actividades cotidianas, tomar las cosas con cautela es un principio. Más aún, para aquellos que han explorado las posibilidades de incorporación de las opciones tecnológicas en los procesos educativos, para aquellos que son los actores reales de la evolución de las TIC en las instituciones y para quienes vienen emprendiendo los proyectos de innovación educativa, la cautela es más que un principio: es uno de los principales fundamentos. La buena noticia es que no hay panacea: los logros siguen dependiendo de las mismas bases: el conocimiento profundo, el dominio de los elementos implicados, el diseño adaptable, el seguimiento cercano, la evaluación, la mejora, y una dosis necesaria de paciencia, y otra de resistencia a la frustración.

El enfoque bimodal o Blended Learning considera el proceso de enseñanza-aprendizaje presencial apoyado por entornos virtuales. Se caracteriza por la flexibilidad e interactividad, por la utilización de los recursos de información y herramientas tales como: los entornos virtuales de aprendizaje (EVA), el e-mail, el chat, el blog, los foros de discusión, entre otros; permite superar obstáculos -como los del tiempo y lugar-en el desarrollo de trabajos en grupo y en la búsqueda de respuestas y soluciones conjuntas. Este enfoque ha abierto un panorama para el aprendizaje colaborativo entre profesores y estudiantes (Barberà \& Badía, 2006). Podemos encontrar en la literatura un amplio soporte a este tipo de estrategias para promover el aprendizaje en una materia o curso, pero también para desarrollar programas educativos completos (Garrison \& Kanuka, 2004). Otros nombres con los que también se le conoce a este enfoque son: híbrido, mixto, de aprendizaje semipresencial, de aprendizaje distribuido, de aprendizaje bimodal o de aprendizaje combinado.

Los estudiantes, al trabajar bajo un enfoque bimodal tienen una mayor flexibilidad de su tiempo y mejoran sus aprendizajes, pero asimismo se encuentran con dificultades para administrar su tiempo, debido a la responsabilidad de gestionar su formación y por la exigencia que les representa tener que usar TIC más especializadas; sin embargo, en los cursos bimodales se propicia una mayor interacción profesor-estudiante, los estudiantes son más participativos trabajando en entornos más flexibles de enseñanza, así mismo tienen más oportunidades para mejorar (Sunkel, Trucco \& Möller, 2011). Al respecto, Garrison \& Kanuka (2004), además de identificar en el enfoque bimodal un gran potencial para combinarlo con formas de aprendizaje profundo y significativo, Profesionalización de promotores de lectura con el aprendizaje basado en proyectos mediado por TIC. Mario Miguel Ojeda Ramírez y Antonia Oliva Jarvio Fernández. 
reconocieron que este enfoque es consistente con los sistemas de valores de las instituciones de educación superior tradicionales, demostrando que estas prácticas pueden mejorar la eficacia y eficiencia de la educación.

Díaz-Barriga \& Hernández-Rojas (2003) analizaron una variedad de enfoques y modelos centrados en el aprendizaje experiencial y significativo, destacando aquellos que centran la construcción del conocimiento en contextos reales y su aplicación dentro de la comunidad fomentando el pensamiento creativo, crítico y reflexivo; algunas de estas estrategias son: Aprendizaje basado en proyectos (ABP), Aprendizaje centrado en la solución de problemas auténticos, Análisis de casos (case method), Prácticas situadas o aprendizaje en escenarios reales, Aprendizaje en el servicio (service learning), Trabajo en equipos cooperativos y Aprendizaje mediado por TIC.

Son este tipo de estrategias las que permiten lograr aprendizajes significativos, aquellos que logran dotar al estudiante de herramientas para pensar, que le permitan el aprendizaje de significados e ideas, acompañadas de fuertes dosis de estrategias cognitivas, metacognitivas, autorreguladoras y crítico-reflexivas; recomiendan el enfoque constructivista y señalan que en diversos estudios las estrategias autorreguladoras (la propia planificación, autosupervisión, autoevaluación, etcétera) tuvieron un resultado favorable en diversos dominios, sobre todo cuando se trató de solucionar problemas reales (Díaz-Barriga, 2010). También a las estrategias autoreguladoras se han sumado posturas socioculturales relativas a los procedimientos en forma situada, no descontextualizada, que implica lograr condiciones reales, metas establecidas, aprovechar recursos internos y externos disponibles, instrumentos que mediaticen la información nueva y dinámicas que permitan la interacción con otros (Belmont, 1989; Wertsch, 1999; Pozo, Monereo \& Castelló, 2001).

Es así que el ABP al hacer uso de estas pedagogías activas, contempla formas de trabajo donde el estudiante tiene que diseñar, aplicar y evaluar un plan para la resolución de una tarea real de tipo profesional -aunque en alguna medida la problemática puede ser simulada- resultando en un conjunto de estrategias en las que el estudio y trabajo personal del estudiante es el eje del proceso formativo, ya que es él quien, bajo la supervisión del profesor, debe ejecutar toda la actividad: construir el marco referencia, revisar las teorías, delimitar el problema, plantear los objetivos, justificar el proyecto y diseñar e implementar la metodología para resolverlo; obtiene las evidencias, al ejecutar las actividades del proyecto, realiza las demostraciones, verificar las soluciones, capta, registra y analiza las evidencias, evalúa los resultados y obtiene conclusiones y recomendaciones. Los proyectos deben surgir del interés de los estudiantes dado que los proponen ellos. Así, lo que aprenden significa mucho para ellos y tiene utilidad, puesto que están inmersos en la construcción de su propio conocimiento; tienen curiosidad y ello les lleva al deseo de investigar, de descubrir, de compartir, de hacerse protagonistas de su propio aprendizaje (Guisasola \& Garmendia, 2014; Larmer \& Mergendoller, 2010; Blumenfeld, Soloway, Marx, Krajcik, Guzdial \& Palincsar, 1991).

$\mathrm{ABP}$ es la suma de actividades que abonan a un solo proyecto que tiene como finalidad resolver una cuestión, tema o conflicto de índole científico o social, a través del involucramiento del estudiante en el diseño y organización en la investigación de su proyecto y en la planificación autónoma de su propio aprendizaje, para culminar en un producto final que presenta ante los demás y que deberá dar soluciones en la realidad. Al respecto el ABP no es considerado parte del currículum, es el currículum en sí Profesionalización de promotores de lectura con el aprendizaje basado en proyectos mediado por TIC. Mario Miguel Ojeda Ramírez y Antonia Oliva Jarvio Fernández. 
mismo, ya que el estudiante se centra en el diseño de su proyecto, no tan solo en cumplir objetivos curriculares. Para el estudiante el conocimiento no se fragmenta pues todas las asignaturas convergen y alimentan el proyecto, por lo que se transfieren o transversalizan los aprendizajes. El ABP Coloca al estudiante en situaciones que lo conducen a recuperar, comprender y aplicar los aprendizajes logrados (Gutiérrez 2003), y resulta más fácil para los maestros trabajar colaborativamente y también evaluar los proyectos de forma conjunta.

Se ha señalado que el ABP permite al docente transmitir de una manera más rápida la información a muchos alumnos a la vez (Reverte, Gallego, Molina, \& Satorre, 2007), e incluso facilita maneras de trabajo colaborativas, ya que los docentes pueden dar seminarios o clases de forma conjunta incluso con estudiantes de otros programas o líneas de investigación. Asimismo, con presentaciones periódicas de sus avances a nivel grupal, los estudiantes fomentan habilidades comunicativas; más aún, los estudiantes hacen un uso más intensivo de la expresión oral y escrita, mejorando en este sentido. Entre estudiantes se desarrollan habilidades colaborativas, incluso sin proponerlo en la planeación, como compartir información, bibliografía, formas de organización y trabajo, etc. En presentaciones de avances de proyectos aprenden a evaluar el trabajo de sus pares y a dar retroalimentación constructiva que los beneficia en ambos sentidos, ya que incentiva la capacidad de autoevaluación y también conduce a un aprendizaje más autónomo (Bará \& Valero, 2006). También existe un mayor trabajo colaborativo entre maestros y estudiantes; los estudiantes aprenden de sus errores al enfrentar y superar retos difíciles e inesperados. Además de que se facilita la utilización de TIC (Barberà, 2006). Sin embargo, el modelo requiere en los estudiantes una planificación y organización del tiempo de manera eficaz y eficiente, y si el tema del proyecto no le es impuesto al estudiante, se traduce en mayor motivación y persistencia en la conclusión.

El ABP mediado por TIC representa un binomio estratégico, por un lado las TIC resultan importantes enlaces que propician fortalezas en el ABP, representan la suma de herramientas digitales que facilitan y sintetizan los procesos de investigación que se llevan a cabo en el desarrollo de proyectos, situación que influye directamente en mejorar las prácticas sobre el aprendizaje, por otro lado el uso de TIC es propio de esta estrategia, favoreciendo el codesarrollo y destacando el hecho de que esta estrategia de aprendizaje es una síntesis de los conceptos y procesos de investigación que influyen directamente en mejorar las prácticas sobre el aprendizaje. La estrategia los beneficia y las herramientas acrecientan las habilidades tecnológicas para convertirlos en comunicadores competentes y solucionadores de problemas avanzados; en ciudadanos de la era del conocimiento (Bell, 2005). La importancia de la utilización de las TIC en el ABP, radica en la necesidad de mejorar la calidad de la educación. "La habilidad más importante de la era digital que deben adquirir los estudiantes es la de aprender a aprender." (Martí, Heydrich, Rojas \& Hernández, 2010, p.12).

Este trabajo recoge las recomendaciones para las buenas prácticas con TIC y las estrategias de aprendizaje aplicadas en el diseño del programa de posgrado de la Especialización en Promoción de la Lectura (EPL) de la Universidad Veracruzana (UV). Es de gran interés que este programa educativo sea exitoso, debido a que en México los índices de lectura son muy bajos, lo que impacta de manera negativa en las expectativas de desarrollo del país. Como lo demuestran las cuatro encuestas se han aplicado en los últimos diez años a nivel nacional, la última fue realizada en el año 2015 y los resultados no son nada alentadores; hay un déficit de lectura en todos los niveles Profesionalización de promotores de lectura con el aprendizaje basado en proyectos mediado por TIC. Mario Miguel Ojeda Ramírez y Antonia Oliva Jarvio Fernández.

Página 4 de 15 
educativos, incluidos los de nivel superior. Por tal motivo, se reconoce que las instituciones educativas tienen un papel preponderante como generadoras e impulsoras del cambio; en este sentido, en la UV se trabaja para mantener un papel destacado en la propuesta y desarrollo de actividades para la promoción de la lectura y la escritura, realizando actividades tales como: programas educativos, festivales culturales, ferias del libro, concursos, clubes de lectura, presentaciones de libros y escritores, portales dedicados a la lectura y la cultura. Esta institución de educación superior pública también cuenta con una editorial, con más de 50 años de tradición literaria y un amplio catálogo de publicaciones; una red estatal de 57 bibliotecas y una biblioteca virtual; la UV es miembro de la Red Internacional de Universidades Lectoras (www.universidadeslectoras.org), cuenta con el programa "Martes de Lectores y Lecturas", "Miércoles de Música y Literatura" y, entre todos estos esfuerzos y logros, destaca la creación de la EPL, único programa de posgrado para la profesionalización de promotores de lectura en México (Jarvio, 2011).

A continuación se presentan el contexto y los antecedentes que dieron lugar a la EPL, se describe, el esquema de diseño curricular y las estrategias de implementación del plan de estudios. Concretamente se analiza la implementación de las experiencias educativas de Taller de Proyecto Integrador I y Proyecto Integrador II, que son el eje de formación del que parte la estrategia del ABP. Se hace referencia a la forma de uso de la plataforma institucional EMINUS para el aprendizaje distribuido, destacando la utilización de bitácoras y entregables asociados al diseño, implementación y elaboración de reportes de proyectos de promoción de la lectura. Se analizan los resultados de las dos primeras generaciones, identificando las áreas de oportunidad de mejora de este sistema de formación basado en un esquema de aprendizaje personalizado que utiliza intensivamente las TIC.

\section{El contexto de la experiencia que se reporta}

Según el último censo mexicano, el de 2010, había entonces 36.9 millones de mexicanos de quince años o más; un grupo donde la inmensa mayoría (93\% de alfabetizados) ya debía ser capaz de leer y escribir; es decir, los alfabetos eran 34.3 millones. Por otra parte, de acuerdo con la Encuesta Nacional de Lectura (CONACULTA, 2006), 11.4\% de los mexicanos alfabetos eran "lectores frecuentes de publicaciones diversas". Esto representa apenas 3.9 millones de lectores, por lo que se deduce que hay más 30.4 millones de alfabetos no lectores en este grupo; es decir, personas que habían aprendido a leer y escribir, pero que no lo hacen más que por razones utilitarias. Definitivamente son muy pocos lectores, sobre todo en relación con lo que hace falta al país para el desarrollo.

Se sabe que los países que invierten mayores porcentajes de recursos en investigación científica son por consiguiente los que cuentan con una mayor producción de conocimiento, así como los que reportan índices y competencias de lectura más elevados, según lo establecen organismos como la Organización para la Cooperación y el Desarrollo Económico (OCDE), El Banco Mundial y los informes PISA; es decir, hay una relación directa entre el nivel de lectura de la población y nivel de desarrollo de un país.

En el último tercio del siglo $\mathrm{XX}$ el reto de la educación mexicana era lograr que la mayoría supiera leer y escribir; ahora hace falta transformar en lectores a esos treinta

Profesionalización de promotores de lectura con el aprendizaje basado en proyectos mediado por TIC. Mario Miguel Ojeda Ramírez y Antonia Oliva Jarvio Fernández. 
millones de alfabetos no lectores. Una institución importante en esta tarea es la escuela mientras no asuma su rol, jamás tendremos una población mayoritariamente lectora-, pero se llevará mucho tiempo abatir el rezago que se padece como país. Para entender, haciendo proyecciones en el corto plazo, hacia 2025 la población lectora sería de 6.1 millones, y la población alfabetizada, pero no lectora ascendería a 36.5 millones. Ante tal reto la pregunta surge de manera natural: ¿quién convertirá en lectores a esos más de treinta millones? Para satisfacer tal necesidad, a lo largo de las tres últimas décadas se han multiplicado los programas de promoción de la lectura, a nivel nacional, estatal, municipal, por iniciativa de instituciones educativas o culturales, y también por parte de particulares y asociaciones civiles. Todos estos programas incluyen un buen número de promotores de la lectura, muchos de los cuales requieren de ser profesionalizados para ser más efectivos en su labor.

Lo anterior no considera la aparición de las TIC y las nuevas formas de lectura, en nuevos soportes, como el libro electrónico, la tableta electrónica, Smartphone, etc., que se alejan de la lectura lineal, lenta y reflexiva que exige el texto impreso, y abren los horizontes a nuevas formas de lectura. De aquí la importancia de considerar el estudio de estas nuevas expresiones comunicativas y de lectura asociadas al uso de los nuevos soportes digitales, y de que esto sea un tema a considerar por los promotores de lectura.

Este es el reto general al que se enfrentan las IES: la formación integral de agentes sociales de cambio: profesionales competentes, ciudadanos plenos en el ejercicio de sus responsabilidades y derechos, promotores de una cultura de valores y humanismo, comprometidos con las mejores causas: el multiculturalismo, la sustentabilidad y la responsabilidad social. Este es el desafío que en nuestros días ha orientado las reformas curriculares de las profesiones y carreras técnicas, y es también lo que ha motivado la creación de nuevos programas académicos: técnico superior universitario, licenciatura, y sobre todo de posgrados, orientados a la investigación y a la profesionalización. Es en este contexto que surge la EPL.

La UV es una universidad pública que atiende aproximadamente a 50 mil estudiantes, alrededor del $30 \%$ de la matrícula de educación superior de Veracruz, México. Está distribuida en 26 ciudades, comprendiendo sus cinco regiones universitarias: Poza RicaTuxpan, Coatzacoalcos-Minatitlán, Córdoba-Orizaba, Veracruz y Xalapa. Aproximadamente el $50 \%$ de la comunidad universitaria se asienta en la ciudad de Xalapa, capital de Veracruz. Cuenta con algunos programas de técnico superior universitario, con más de 50 licenciaturas -en todas las áreas del conocimiento- y con más de 100 programas de posgrado: de especialización, maestría y doctorado.

Una de las estrategias aplicadas en la última década para el desarrollo institucional de la UV se basó en la adopción de los principios, políticas y estándares de calidad del Programa Nacional de Posgrados de Calidad (PNPC), programa del Consejo Nacional de Ciencia y Tecnología (CONACYT) y de la Secretaría de Educación Pública (SEP). La forma de trabajo y los procedimientos seguidos, así como los logros obtenidos se han reportado recientemente (Cruz-González, 2016). Fruto de estos esfuerzos es la EPL, posgrado que surge del Programa Universitario de Formación de Lectores (PUFL); la aprobación del plan de estudios por órganos colegiados institucionales se dio en el 2013, iniciando cursos en el 2014. El programa se diseñó y desarrolló acorde a los principios y lineamientos del PNPC, donde en la evaluación resultó exitosa. Este proceso ha sido documentado en una memoria (Zamora-Barragán, 2015).

Profesionalización de promotores de lectura con el aprendizaje basado en proyectos mediado por TIC. Mario Miguel Ojeda Ramírez y Antonia Oliva Jarvio Fernández. 
Esta especialización, única en el país, será un importante apoyo: se constituye en un espacio académico generador de promotores de lectura con bases sólidas para desarrollar su labor de forma profesional. Destaca no sólo por su enfoque de formación, sino también porque utiliza intensivamente las TIC para llevar a cabo procesos educativos de forma guiada. La EPL cuenta ahora ya con tres sedes: Xalapa, Córdoba y Veracruz (www.uv.mx/epl).

\section{Diseño curricular de la EPL y el proceso de implantación}

El currículum de la EPL se centra en el ABP. A tal fin, los estudiantes son seleccionados a partir de una propuesta de un proyecto de promoción de lectura, para lo que se proporciona una guía. Lo que se propone es un proyecto de intervención (talleres, programas, círculos creación de espacios, campañas, etc.) para promover la lectura en grupos específicos o a través de las nuevas formas de lectura en la era digital (Cordón y Jarvio, 2015). En el proceso de admisión se valoran las competencias de los aspirantes (nivel lector, escritura, experiencia en promoción de la lectura, uso de TIC, claridad de objetivos, dominio del proyecto y motivación), ya que se permite la certificación de competencias, lo que hace que se pueda diseñar para cada estudiante un plan personalizado (Zamora-Barragán, 2015). Los estudiantes inician actividades con una carga de hasta 7 cursos: Lectura y escritura en el contexto universitario, Cartografías lectoras, Lectura y escritura tras las líneas, Estrategias de promoción y fomento de la lectura, Taller de lectura en voz alta, dos optativas y Proyecto Integrador I (ver Tabla 1). En el marco de Proyecto Integrador I, cada estudiante lleva un registro programado en una bitácora en línea, donde planifica sus actividades con herramientas de calendarización, reportando las actividades y las contingencias (Sarur y Ojeda, 2016). Desde el primer momento el estudiante se incorpora a un sistema de tutorías, presenciales y en línea, que tienen la finalidad de guiarlo y acompañarlo durante su proceso formativo, en una primera instancia con un tutor, y una vez que se ha aprobado el protocolo de su proyecto, a partir de un comité tutorial. Las actividades que se realizan en el taller de Proyecto Integrador I, y de las otras materias, le dan los elementos metodológicos y disciplinares para la elaboración de un protocolo de un proyecto de intervención acorde a estándares bien establecidos, para lo cual se cuenta con una guía y el apoyo del tutor y los profesores de los cursos; en este periodo es fundamental durante las sesiones presenciales del enfoque bimodal, el seguimiento y retroalimentación del coordinador del taller, a través de reportes de lectura, mapas conceptuales, ensayos, bitácoras y presentaciones de sus protocolos (marco referencial, delimitación del problema, objetivos, justificación, estrategia de intervención, metodología de evaluación y cronograma). Cabe destacar que los protocolos son el eje de una formación personalizada considerando estrategias de trabajo colaborativo a partir de un plan al que le da seguimiento el comité tutorial (www.uv.mx/epl/generaciones) en el marco del segundo taller: Proyecto Integrador II. 
Tabla no 1.

Estructura del plan de estudios de la EPL.

\begin{tabular}{|l|c|c|c|}
\hline \multirow{2}{*}{ Nombre de la experiencia educativa } & \multirow{2}{*}{ Créditos } & \multicolumn{2}{|c|}{ Horas } \\
\cline { 3 - 4 } & & Teoría & Práctica \\
\hline 1. Lectura y escritura en el contexto universitario & 5 & 30 & 15 \\
\hline 2. Cartografías lectoras & 5 & 15 & 45 \\
\hline 3. Lectura y escritura tras las líneas & 5 & 30 & 15 \\
\hline 4. Estrategias de promoción y fomento de la lectura & 4 & 15 & 30 \\
\hline 5. Escritura y lectura de la era analógica a la era digital & 4 & 15 & 30 \\
\hline 6. Taller de géneros de lectura y escritura & 4 & 15 & 30 \\
\hline 7. Seminario de metodología de la lectura & 5 & 30 & 15 \\
\hline 8. Literatura y otras manifestaciones culturales & 4 & 15 & 30 \\
\hline 9. Taller de lectura en voz alta & 4 & 15 & 30 \\
\hline 10. Proyecto integrador I & 4 & 15 & 30 \\
\hline 11. Proyecto integrador II & 4 & 15 & 30 \\
\hline 12. Optativa I & 3 & 15 & 15 \\
\hline 13. Optativa II & 3 & 15 & 15 \\
\hline 14. Optativa III & 3 & 0 & 30 \\
\hline 15. Optativa IV & 3 & 0 & 30 \\
\hline Total & 60 & 240 & 390 \\
\hline
\end{tabular}

Con las estrategias de seguimiento personalizado los estudiantes de la EPL, hacen investigación documental, revisan reportes y experiencias similares de su proyecto de intervención, diseñan una estrategia metodológica para la intervención y para la evaluación de los resultados, así como implementan y evalúan las acciones de promoción de la lectura enmarcadas en su proyecto. Se pretende que a partir del ABP los estudiantes aprendan a conducir su aprendizaje; busquen información y la procesen adecuadamente; posean estrategias de organización y análisis; planifiquen su aprendizaje; trabajen colaborativamente; opinen sobre avances de los trabajos y exposiciones de sus compañeros (considerado como parte del trabajo colaborativo); y compartan información, como bibliografía o instrumentos de evaluación, así como estrategias metodológicas; todo esto con el apoyo de tutorías oportunas.

La función del modelo de ABP mediado por TIC es la de integrar los saberes mediante estrategias de aprendizaje, además de guiar y darle seguimiento no sólo al estudiante, también a los profesores en sus procesos de enseñanza y de aprendizaje durante el año que dura el programa educativo; en este sentido, en la parte que corresponde al trabajo llevado a cabo en línea se hace uso de EMINUS, el Entorno Virtual de Aprendizaje (EVA) de la Universidad Veracruzana, definido por sus desarrolladores de la siguiente manera:

Eminus es un sistema de administración de ambientes flexibles de aprendizaje el cual sirve para presentar cursos en línea para distribuirse en Internet o redes internas. Permite la comunicación en forma sincrónica y asincrónica ya que utiliza las tecnologías de la información y la comunicación para aprovechar la facilidad de distribución de materiales formativos y herramientas de comunicación, lo que permite

Profesionalización de promotores de lectura con el aprendizaje basado en proyectos mediado por TIC. Mario Miguel Ojeda Ramírez y Antonia Oliva Jarvio Fernández. 
crear un entorno completo para el aprendizaje ayudando a la vez a mejorar los niveles educativos sin límites de tiempo y de distancia, permitiendo a cada estudiante tomar el control de su aprendizaje y formación de una forma independiente y colaborativa. Con este sistema se redefine la docencia de manera más placentera, útil y eficiente con énfasis en la comunicación, la colaboración y la distribución de materiales de enseñanza y aprendizaje (Colunga y Jiménez, 2007, p.7).

En EMINUS los estudiantes reportan su programa de trabajo y avances formativos semanalmente a través de una bitácora; en ella se incluyen dos grandes apartados: (1) reporte de las actividades, tiempos de dedicación, contingencias y observaciones; el reporte se hace diariamente y al final de la semana se realiza una revisión y se aplica una autoevaluación, identificando los retos y pendientes para (2) la planeación de la semana siguiente; conforme al plan de estudios -las actividades de los cursos y las propias del proyecto-, programando los tiempos de dedicación y estableciendo una lista de prioridades. Para evaluar y dar un seguimiento preciso a la bitácora se establecieron criterios, procedimientos e indicadores de evaluación: redacción, pertinencia, planificación y completitud, asignándoles puntajes y definiendo una categoría de semaforización. Desde un inicio se revelaron las dificultades en algunos estudiantes, principalmente en la organización del tiempo y la información, así como algunas carencias formativas.

Al respecto Solórzano (2009), afirma que los estudiantes presentan dificultades con la cantidad de información especializada a la que acceden debido a que no saben cómo administrarla, procesarla ni ordenarla gradualmente para después darle un sentido a lo que aprenden, es decir, no reconocen por qué y para qué lo aprenden en función del momento educativo en el que se encuentran inmersos, detectando un impacto negativo, no en la falta de recursos materiales, como muchas veces se cree, sino en el aburrimiento generado por la dispersión y el derroche de esfuerzos.

En este punto, una de las mayores dificultades que se identificó en los estudiantes, además de la gran cantidad de información que acumularon y el tiempo excesivo que destinaron a su búsqueda, fue el no saber establecer límites y alcances sobre la cantidad de información y el tiempo destinado para su recopilación, datos que en un inicio no reportaron en sus bitácoras. Hay que señalar que para la EPL, la planeación de estas actividades resulta tan importante como el hecho de que sepan buscar información valiosa, pertinente y de fuentes confiables. Esto se pudo constatar en un inicio con la revisión y evaluación de sus bitácoras. Ver Figura 1. 


\section{Revisión del facilitador}



Figura 1. Ejemplo de retroalimentación a la bitácora correspondiente a la semana 1.

Se observó que los estudiantes que se adaptaron rápidamente a los requerimientos de la bitácora (3 de 19) tuvieron una mayor ventaja académica sobre aquellos que mostraron resistencia para hacerla (7 de 19); estos últimos, en sus propias palabras, se resistían porque consideraban que la bitácora sólo era "una carta de buenas intenciones".

Encontramos varios estudiantes que adolecían de una cultura de la planificación y programación de su tiempo, pero gradualmente se fueron adaptando a los requerimientos; ponemos como ejemplo una trayectoria de desempeños (ver Figura 2), donde claramente vemos un puntaje creciente, durante catorce semanas realizando la bitácora; cabe destacar que esta estudiante concluyó sus estudios obteniendo altos rendimientos y una nota laudatoria en su examen para obtener el diploma de la especialización.

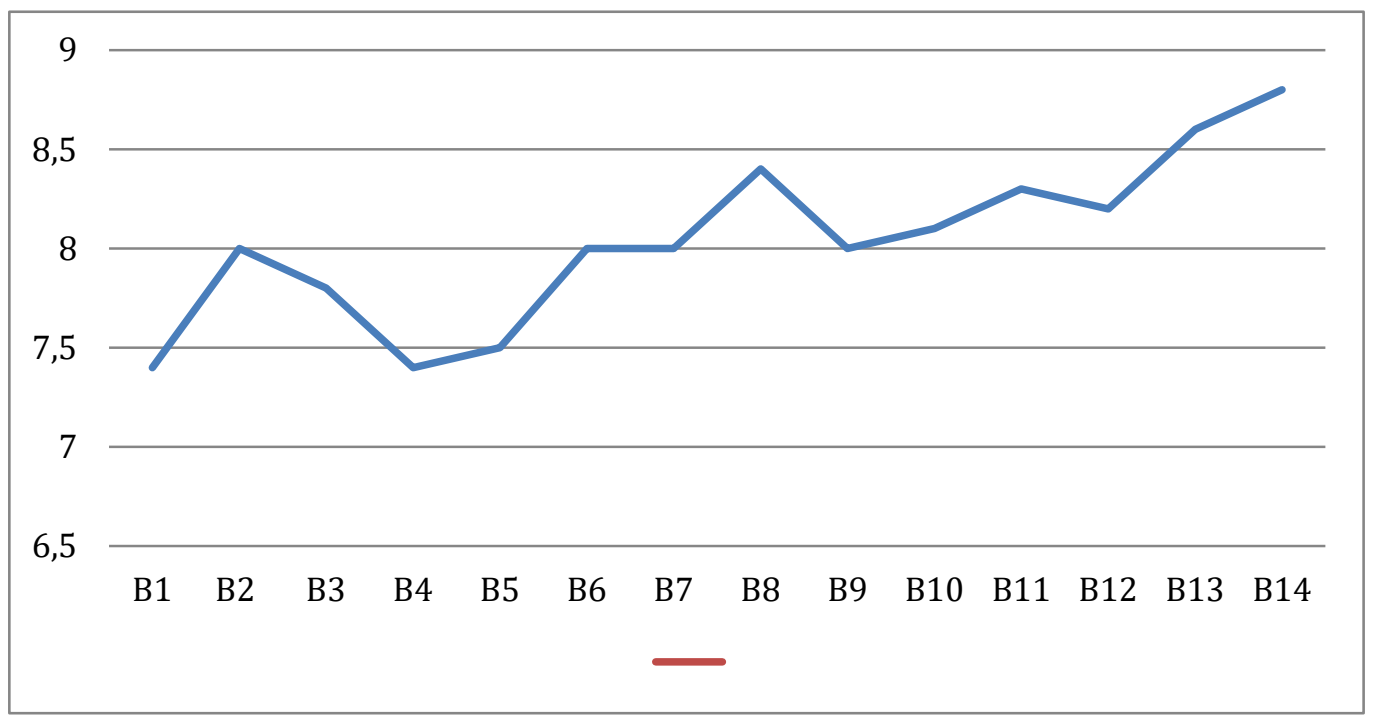

Figura 2. Estudiante con una evolución positiva en el uso de la bitácora.

Profesionalización de promotores de lectura con el aprendizaje basado en proyectos mediado por TIC. Mario Miguel Ojeda Ramírez y Antonia Oliva Jarvio Fernández. 
Existe la idea equivocada de que programar con precisión los tiempos y actividades se convierte para ellos en un compromiso estricto e inflexible. Si bien es cierto que no todo aquello que es programado en la bitácora se realiza en la fecha señalada, hay que destacar que el evadir la definición de un tiempo para sus actividades no les permite saber en qué punto de su proceso educativo están situados, con el riesgo de desconocer qué han realizado, en dónde se encuentran y qué les falta por hacer. Ignorar estos aspectos tan esenciales, genera ansiedad e incertidumbre; esto último fue reconocido por aquellos que adoptaron el enfoque de autoevaluación y planeación, que como puede verse en la Figura 3 fueron en su mayoría aquellos con un positivo desempeño en las bitácoras, obteniendo mejores rendimientos totales $(r=0.40, \mathrm{p}<0.001)$.



Figura 3. Asociación entre rendimiento total y calificación en la bitácora.

\section{Oportunidades de mejora y conclusiones}

No podemos dejar de considerar el valor que los estudiantes le dan a las sesiones presenciales, pero en la medida en que se habitúan al enfoque bimodal y al uso de los recursos de la plataforma EMINUS se percibe claramente que se adaptan a las nuevas ventajas de tener en un sólo lugar una serie de recursos de información y de organización. El ABP requiere gran cantidad de tiempo disponible para realizar las actividades, las cuales en un principio requieren de la comprensión profunda de lecturas académicas, para posteriormente elaborar reportes de lectura y realizar mapas conceptuales que implican un trabajo individualizado, aunque se compartan estrategias e inclusive lecturas, cada proyecto tendrá sus necesidades particulares. El trabajo creativo e independiente hace que los estudiantes aprecien las TIC, ya que ante cualquier duda pueden consultar al tutor y a los académicos a través de diversos canales de comunicación en línea. En la medida en que la programación del uso del tiempo destinada a las actividades del proyecto y de las materias toman prioridad, los 
estudiantes se mueven con más soltura en su proceso de formación, y tienen un mayor rendimiento.

El primer reto a vencer en los estudiantes es lograr el convencimiento de que no hay fórmulas mágicas, que lo que siempre paga es la dedicación, el trabajo de calidad, la concentración y las buenas prácticas de estudio. Los estudiantes de posgrado son, en general, maduros y tienen agendas muy visibles. En el caso de la EPL se encuentran dedicados de tiempo completo a su proceso formativo, considerando las reglas de las becas de manutención otorgadas en este programa, hacen que sea difícil que se muevan hacia agendas ocultas, lo que indudablemente deja en evidencia los desempeños académicos de los estudiantes.

Los casos que ocasionaron baja, y aquellos que no han concluido aún su trabajo recepcional muestran que el sistema formativo de la EPL es de máxima exigencia, y que una evasión de su planeación o no presentarse a una evaluación ocasionan problemas de impacto en el rendimiento. Aunque se tiene un caso que es la excepción, ya que aunque no logró los mejores rendimientos sí concluyó con el éxito el proyecto y obtuvo el diploma. Lo que lo distingue son sus competencias en el uso de las TIC.

La admisión a la EPL debe elevar sus niveles de rigurosidad, ya que hay competencias en la lectura y la escritura académicas que de no contar con ellas el tránsito por el proceso formativo se hace muy riesgoso.

Para finalizar, concluimos que dentro de la EPL se reconoce la necesidad de seguir elaborando recursos de apoyo digitales (tutoriales, guías, videos, etc.) que ayuden en el diseño, desarrollo, evaluación y elaboración de los protocolos y reportes, y que a la vez estos materiales puedan ser consultados en todo momento con la ayuda de Eminus. También se debe afianzar la coordinación de los profesores y la articulación de los contenidos de las materias; mejorar los procesos de evaluación de las intervenciones y fortalecer el sistema tutorial presencial y en línea. El rumbo es el correcto, pero aún queda mucho camino.

Agradecimientos: Agradecemos en primer lugar a Belinda Sarur, quien como parte de su formación en la Maestría en Educación Virtual de la Universidad Veracruzana, realizó un proyecto de tesis del que se deriva este trabajo. También a los árbitros y colegas que hicieron sugerencias de mejoras sobre versiones preliminares de este artículo, el cual fue presentado como una ponencia en el XI International Guide Conference and IX International Edtech Ikasnabar Congress e-learning 2016: new strategies and trends, en Madrid los días 22 al 24 de junio.

Presentación del artículo: 13 de febrero de 2017 Fecha de aprobación: 15 de marzo de 2017 Fecha de publicación: 31 de marzo de 2017 
Ojeda Ramírez, M. M., Jarvio Fernández, A. O., \& Sarur Larrinaga, B. (2017). Profesionalización de promotores de lectura con el aprendizaje basado en proyectos mediado por TIC. RED. Revista de Educación a Distancia, 54. Consultado el (dd/mm/aaaa) en http://www.um.es/ead/red/54/ojeda_jarvio.pdf

\section{Financiación}

Esta investigación no ha recibido ninguna subvención específica de los organismos de financiación en los sectores públicos, comerciales o sin fines de lucro.

\section{Referencias bibliográficas}

Bará, J. Valero, M. (2006). Taller de Formación: Aprendizaje Basado en Proyectos. Universitat Politècnica de Catalunya: España.

Barberà, E. y Badía, A. (2005). El uso educativo de las aulas virtuales emergentes en la educación superior. Revista de Universidad y Sociedad del Conocimiento. 2(2). España: UOC. Recuperado de: http://www.uoc.edu/rusc/2/2/dt/esp/barbera.pdf

Barberà, E. (2006). Aportaciones de la tecnología a la e-Evaluación. Revista de Educación a Distancia. Recuperado de: http://www.um.es/ead/red/M6.

Bell, S. (2005) Project-Based Learning for the 21st Century: Skills for the Future. The Clearing House, 83, 39-43. Taylor \& Francis Group. Recuperado de: http://dx.doi.org/10.1080/00098650903505415

Blumenfeld, P., Soloway, E., Marx, R., Krajcik, J., Guzdial, M., y Palincsar, A. (1991). Motivating project-based learning: Sustaining the doing, supporting the learning. Educational Psychologist, 26(3-4), 369-398.

Belmont, J. M. (1989). Las estrategias congnitivas y de aprendizaje estratégico: El enfoque socio-educacional. American Psychologist. 44(2),142-148. http://dx.doi.org/10.1037/0003-066X.44.2.142

Colunga, J. A. y Jiménez, J. C., (2007). Eminus. Sistema de Educación Distribuida. La Escuel@ del Futuro. Conferencia presentada en Virtual Educa (Brasil). http://reposital.cuaed.unam.mx:8080/jspui/bitstream/123456789/1169/1/16JCM.PDF

CONACULTA (2006). Encuesta Nacional de Lectura. México: Autor.

Cordón, J. A. y Jarvio, A. O. (2015). ¿Se está transformando la lectura y la escritura en la era digital? Revista Interamericana de Bibliotecología, 38(2), 137-145. DOI:10.17533/udea.rib.v38n2a05.

Cruz-González, G. (2016). La Planeación Estratégica para el Aseguramiento de la Calidad en el Posgrado de la Universidad Veracruzana, Periodo 2009-2014. Tesis de Maestría en Gestión de la Calidad, Universidad Veracruzana: México. (www.uv.mx/gestión/generaciones).

Profesionalización de promotores de lectura con el aprendizaje basado en proyectos mediado por TIC. Mario Miguel Ojeda Ramírez y Antonia Oliva Jarvio Fernández. 
Garrison, R. y Kanuka, H. (2004). Blended learning: Uncovering its transformative potential in higher education. Canada: Elsevier.

Guisasola, J. y Garmendia, M. (Eds.) (2014). Aprendizaje basado en problemas, proyectos y casos: diseño e implementación de experiencias en la universidad. Servicio Editorial de la Universidad del PaísVasco: España.

Gutiérrez, O. (2003). Enfoques y modelos educativos centrados en el aprendizaje. Estado del arte y propuestas para su operativización en las Instituciones de Educación Superior nacionales. En Métodos y estrategias para favorecer el aprendizaje en las Instituciones de Educación Superior. Documento 3. Universidad Politécnica Nacional. México. Recuperado de: http://www.lie.upn.mx/docs/docinteres/EnfoquesyModelosEducativos3.pdf

Díaz-Barriga, F. (2010). Los profesores ante las innovaciones curriculares. Revista Iberoamericana de Educación Superior 1(1), 37-57. Recuperado de: https://ries.universia.net/article/viewFile/32/91

Díaz-Barriga, F. y Hernández-Rojas, G. (2003). Estrategias Docentes para un Aprendizaje Significativo. Una interpretación constructivista. En: Estrategias para el aprendizaje significativo: fundamentos, adquisión y enseñanza. México: Mc Graw Hill.

Jarvio, A. O. (2011). La lectura digital en el ámbito de la Universidad Veracruzana (Vol. 282). Universidad de Salamanca.

Larmer, J. y Mergendoller, J. (2010). 7 Essentials for project-based learning. Educational Leadership, 68(1), 34-37.

Martí, J., Heydrich, M., Rojas, M. \& Hernández, A.(2010). Aprendizaje basado en proyectos: una experiencia de innovación docente. Revista Universidad EAFIT. 46(158),11-21. Medellín, Colombia: Universidad EAFIT.

Pozo., J. I., Monereo, C. y Castelló, M. (2001). El uso estratégico del conocimiento. En Coll, C., Palacios, J. y Marchesi, A. (Coords.). Psicología de la Educación Escolar. Madrid: Alianza Editorial, 211-258.

Reverte-Bernabeu, J., Gallego-Sáchez, A, Molina-Carmona, R. y Satorre-Cuerda, R. (2007). El Aprendizaje basado en proyectos como modelo docente. Experiencia interdisciplinar y herramientas groupware. http://hdl.handle.net/10045/1808

Ramírez-Martinell, A. y Casillas-Alvarado, M. (2014). Háblame de TIC. Tecnología Digital en la Educación Superior. Argentina: Editorial Brujas.

Sarur, B. y Ojeda, M. M. (2016). Uso del tiempo de los Estudiantes en cursos universitarios Mediados por Tecnologías de la Información y la Comunicación. En Casillas, M. y Ramírez-Martinell, A. (Coords.). Háblame de TIC 3: Educación Virtual y Recursos Informativos. Argentina: Editorial Brujas, 185203.

Profesionalización de promotores de lectura con el aprendizaje basado en proyectos mediado por TIC. Mario Miguel Ojeda Ramírez y Antonia Oliva Jarvio Fernández. 
Solórzano, C. (2009). Estrategias metodológicas para su aplicación en el aula. En Solórzano, C. \& Valverde, K. (Coords.) (2009). Experiencias docentes en ciencias sociales para la educación superior. México: UNAM.

Sunkel, G. Trucco, D. y Möller, S. (2011). Aprender y enseñar con las tecnologías de la información y las comunicaciones en América Latina: potenciales beneficios. Serie Políticas Sociales. No. 169. Santiago de Chile: Publicación de las Naciones Unidas.

Tapscott, D. (2009). La era digital. México: McGraw Hill.

Westch, J. V. (1999). La mente en acción. Buenos Aires: Aique.

Zamora-Barragán, E. L. (2015). Proceso de acreditación de la Especialización en Promoción de la Lectura. Tesis de Maestría en Gestión de la Calidad, Universidad Veracruzana: México. (www.uv.mx/gestion/generaciones). 